\title{
FUNCTORIAL RADICALS AND NON-ABELIAN TORSION
}

\author{
by SHALOM FEIGELSTOCK and AARON KLEIN
}

(Received 1st May 1979)

Radicals appear in many algebraic contents. For modules over a ring, they give rise to pre-torsion and torsion theories, Goldman (5), Lambek (14). In the category of groups, Kurosh, Plotkin and others have introduced radicals $(\mathbf{6}),(\mathbf{1 3}),(\mathbf{2 1})$, but unlike the radicals in module theory these radicals are not necessarily functorial, as for example the nil radical and the Hirsch-Plotkin radical (6). The functorial method in module theory has been extended to abelian categories, Dickson (2), to the category of nilpotent groups, Hilton (8), Warfield (25), and to the category of groups, Plotkin (22), and to general categories, Wiegandt (26), Holcombe and Walker (10).

In this paper the theories for groups and for modules are united by gathering both types of algebraic structures under a wider concept of module, namely not necessarily commutative $(\bigwedge, \Sigma)$-modules, where the set of scalars $\bigwedge$ is a near-ring distributively generated by a monoid $\Sigma$. These are the $(\bigwedge, \Sigma)$-groups of Fröhlich (4).

The non-abelian case has been extensively studied by Barr (1), Lambek and Rattray (15), (16), (17), (23). Their theory makes use of injectives. Lack of injectives in the general case implies that a general theory has to be kept down to a sort of "pre-torsion" and this is done in the present paper. The definitions are for categories of $(\bigwedge, \Sigma)$-modules since we propose a theory which should apply to both groups and modules. Several of the concepts considered here were introduced by Holcombe and Walker in a more general setting.

In Section 1, a theory of radicals and torsion is constructed for $(\bigwedge, \Sigma)$-modules. This approach unites the torsion and pre-torsion theories for modules with the study of radical-classes of groups (for functorial radicals).

In Section 2, which may be viewed as an independent purely group-theoretic contribution, we pause to construct extensions of the torsion and torsion-free functors whose operations on the category of abelian groups are well known. A new concept of "pretorsion group" arises. It seems that this is the right class in the category of groups which extends the class of torsion abelian groups: the pair torsion-abelian - torsion-free abelian extends to pre-torsion - torsion-free which is the pair radical - semisimple for the radical constructed. Important classes of groups are classified with respect to their torsion type. The following is a benefit. In the presentation theory of groups it is useful to have conditions which determine whether or not a given group is a one-relator group. For instance, in constructing groups for which the word problem is unsolvable one must be careful not to select one-relator groups as possible candidates. The radical constructed in Section 2 provides a sufficient condition for a group not to be a one-relator group.

We proceed with a short section to exhibit other radicals for groups. Some important 
classes of groups are realised as either the radical class or the semisimple class associated with radicals.

In the last section we sketch briefly generalities concerning classes of radicals. Further details and ramifications will appear elsewhere. Some points, not approached in this paper (such as the influence of injectives with respect to given classes of monomorphisms, existence of factorisation systems (15), the problem of quotients, topological considerations), are of sufficient interest to deserve further study.

\section{Radicals and torsion}

Let $(\bigwedge, \Sigma)$ stand for a unitary distributively generated rear-ring. So (i) $\langle\wedge,+\rangle$ is a group and $\langle\Lambda, \cdot\rangle$ is a monoid; (ii) $\left(\lambda_{1}+\lambda_{2}\right) \lambda=\lambda_{1} \lambda+\lambda_{2} \lambda$ for all $\lambda_{1}, \lambda_{2}, \lambda \in \Lambda$; (iii) $\langle\Sigma, \cdot\rangle$ is a submonoid of $\langle\Lambda, \cdot\rangle$; (iv) $\Sigma$ is a set of generators for the group $\langle\bigwedge,+\rangle$; and (v) $\sigma\left(\lambda_{1}+\lambda_{2}\right)=$ $\sigma \lambda_{1}+\sigma \lambda_{2}$ for all $\sigma \in \Sigma, \lambda_{1}, \lambda_{2} \in \Lambda$.

We write groups additively, but this is not meant to imply that they are abelian. For groups $X, Y$ denote by $\operatorname{MAP}(X, Y), \operatorname{hom}(X, Y), \operatorname{HOM}(X, Y)$ respectively, the group of mappings of $X$ into $Y$ with natural addition, the set of homorphisms of $X$ into $Y$, the subgroup of $\langle\operatorname{MAP}(X, Y),+\rangle$ generated by hom $(X, Y)$. Consequently $(\operatorname{HOM}(X, X))$, with the natural composition, is a unitary distributively generated near-ring.

Definition 1.1. A $(\bigwedge, \Sigma)$-module $X, m$ consists of a group $\langle X,+\rangle$ with a morphism of distributively generated near-rings $m:(\bigwedge, \Sigma) \rightarrow(\operatorname{HOM}(X, X), \operatorname{hom}(X, X))$, As a rule we write $\lambda x$ for $m(\lambda) x(\lambda \in \Lambda, x \in X)$.

For $(\Lambda, \Sigma)$-modules $X, Y$ we denote by hom $\operatorname{hom}_{\wedge}(X, Y)$ the set of $\wedge$-homomorphisms of $X$ into $Y$ and $\operatorname{HOM}_{\wedge}(X, Y)$ the subgroup of $\langle\operatorname{MAP}(X, Y),+\rangle$ generated by $\operatorname{hom}_{\wedge}(X, Y)$. The respective categories will be denoted by $(\bigwedge, \Sigma)$-mod, $(\bigwedge, \Sigma)$-MOD.

In particular: $(Z,\{1\})$-mod is the category of groups $\mathscr{G}_{r} ;(Z,\{1,-1\})-\mathrm{MOD}=$ $\{Z,\{1,-1\})$-mod is the category of abelian groups $\mathscr{A} b ;(\wedge, \wedge)$-mod $=(\wedge, \wedge)$-MOD with a unitary ring $\Lambda$ is the category of ordinary $\wedge$-modules; $\left(Z[t],\left\{1, t, t^{2}, \ldots\right\}\right)$-mod is the category of pairs $(X, \xi)$ consisting of groups $X$ with a fixed endomorphism $\xi: X \rightarrow X$, and homomorphisms that form commutative squares with the given endomorphisms.

Observe that $K \subset X$ is a kernel in $(\bigwedge, \Sigma)$-mod iff $K$ is a submodule and a normal subgroup of $X$. In this case we shall write $K \triangleleft X$.

Let $\mathscr{A}$ be a full subcategory of $(\bigwedge, \Sigma)$-mod closed under epimorphic images and monomorphic pre-images.

Definition 1.2. A pre-radical on $\mathscr{A}$ is a functor $R: \mathscr{A} \rightarrow \mathscr{A}$ which is a normal subfunctor of the identity of $\mathscr{A}$. A radical is a pre-radical $R$ satisfying $R(X / R(X))=0$ for all $X$. An idempotent (pre-)radical is a (pre-)radical $R$ satisfying $R^{2}=R$.

An idempotent pre-radical on $\mathscr{A}$ evidently gives rise to an idempotent cotriple on $\mathscr{A}$. If $R$ is a radical then $S=I / R$ is an idempotent functor which gives rise to a triple on $\mathscr{A}$. To obtain a torsion theory, $S$ is required to preserve regular monics, Barr (1). In our case this is not satisfied in general (see for instance $S=I / T$ on $\mathscr{G}_{r}, 2.8$ ). Actually in (1) injective effacements are employed, but in $\mathscr{G}_{r}$ there are no non-trivial injective effacements (12).

So a pre-radical $R$ assigns to each object $X$ of $\mathscr{A}$ a submodule $R(X) \triangleleft X$ such that $f(R(X)) \subset R(Y)$ for all $Y$ and $f \in \operatorname{hom}_{\wedge}(X, Y)$. If $\mathscr{A}=(\bigwedge, \Sigma)$-mod then, by additivity, $R$ 
extends to a normal subfunctor of the identity on $(\bigwedge, \Sigma)$-MOD. Unfortunately, a submodule is not necessarily a normal subgroup. To bypass this flaw we add the following assumption in certain cases.

(a) For every $X$, hom ${ }_{\wedge}(X, X)$ includes the group conjugations $x \mapsto^{a} x=a+x-a$ for all $\mathbf{a} \in \mathbf{X}$.

The presence of $(a)$ permits the omission of the word "normal" from Definition 1.2 since $f(R(X)) \subset R(X)$ for all $f \in \operatorname{hom}_{\wedge}(X, X)$, in particular for all the conjugations.

The following statement is equivalent to $(a)$ : In every $X, \sigma a+\sigma x-\sigma a=a+\sigma x-a$ for all $\sigma \in \Sigma, a, x \in X$.

Observe that in the theory for nilpotent groups (25), the objects are $R$-groups for a binomial domain $R$ and they are required to meet two extra conditions; one of them is precisely our $(a)$. (The other is the Hall-Petresco formula, 10.4 (25).)

The assumption (a) obviously holds in the category of groups $(Z,\{1\})$-mod and in categories of modules over rings $(\Lambda, \wedge)$-mod. The following is a further example with $(a)$. The category $\left(Z,\left\{3^{k} \mid k \geqq 0\right\}\right)$-mod is easily shown to be the variety of groups satisfying the law $2 a+2 b=2(b+a)$ and (a) follows from $3 a+3 x-3 a=a+x+2(a+x)-3 a=$ $a+3 x-a$.

The category $\left(Z[t],\left\{t^{k} \mid k \geqq 0\right\}\right)$-mod does not satisfy $(a)$. The category

$$
\left(Z[t],\left\{t^{k}(1+t)^{l} \mid k, l \geqq 0\right\}\right)-\bmod
$$

is precisely the category of pairs $(X, \xi)$ composed of a group $X$, and a central endomorphism $\xi$, and homomorphisms that form commutative squares with the prescribed endomorphism. Indeed, with such a pair $(X, \xi)$ the map $1+\xi$ is an endomorphism and we define $p(t) a=p(\xi) a$ for all $p(t) \in Z[t], a \in X$. Conversely for an object in the above category the map $\xi: a \mapsto t a$ is an endomorphism and it is central since $1+\xi$ has to be an endomorphism. It follows that both $a+t b-a$ and $t a+t b-t a$ are always $t b$ and similarly with $1+t$ instead of $t$. Hence $(a)$ holds in this category. Observe that a non-zero nilpotent group admits non-zero central endomorphisms. (If $X$ is nil- $k$ there are $b_{1}, \ldots, b_{k} \in X$ such that $\left[b_{1}, \ldots, b_{k}\right] \neq 0$. With $b=\left[b_{1}, \ldots, b_{k-r}\right]$, since $\left[a_{1},\left[b, a_{2}\right]\right]=0$ for all $a_{1}, a_{2} \in X$, it follows that $\xi: a \mapsto[b, a]$ is a central non-zero endomorphism on $X$.)

For classes $\mathscr{B}, \mathscr{C}$ of $(\bigwedge, \Sigma)$-modules denote

$$
\mathscr{B}^{\prime}=\left\{Y \mid \operatorname{hom}_{\wedge}(B, Y)=0, \forall B \in \mathscr{B}\right\}, \mathscr{C}^{l}=\left\{X \mid \operatorname{hom}_{\wedge}(X, C)=0, \forall C \in \mathscr{C}\right\} .
$$

Before we state the following theorem let us mention that the categories $(\bigwedge, \Sigma)$-mod admit products and coproducts, (4), (19), If $\mathcal{M}$ is a class of $(\bigwedge, \Sigma$ )-modules, we say that $X$ is an $\mathcal{M}$-extension of $M$ if $M$ and $X / M$ are in $\mathcal{M}$.

Theorem 1.2. Assume that $\mathscr{A}$ is a category with coproducts. The following statements are equivalent for classes $\mathscr{B}, \mathscr{C}$ of objects in $\mathscr{A}$, with (a) assumed: (i) $\mathscr{B}=\mathscr{C}^{l}$ and $\mathscr{C}=\mathscr{B}^{r} ;$ (ii) $B$ is closed under epimorphic images, coproducts, $\mathscr{B}$-extensions and $\mathscr{C}=\mathscr{B} r$; (iii) There exists an idempotent radical $R$ on $\mathscr{A}$ such that $\mathscr{B}=\{X \mid R(X)=X\}$ and $\mathscr{C}=\{X \mid R(X)=0\}$.

Proof. (i) $\Rightarrow$ (ii) and (iii) $\Rightarrow$ (i) are easily verified. To establish (ii) $\Rightarrow$ (iii) consider the set of submodules of $X$ which are in $\mathscr{B}$ and call $R(X)$ the epimorphic image in $X$ of their coproduct. So $R(X) \in \mathscr{B}$ and if $X \in \mathscr{B}$ then $R(X)=X$ by construction, thus $R^{2}=R$. 
Since any $f: X \rightarrow Y$ takes $\mathscr{B}$-submodules onto $\mathscr{B}$-submodules it follows that $f(R(X)) \subset$ $R(Y)$ and $R(X) \triangleleft X$ by $(a)$. The module $X / R(X)$ does not possess non-trivial $\mathscr{B}$ submodules, since $U / R(X) \in \mathscr{B}$ would imply $U \in \mathscr{B}$ so $U \subset R(X)$. Thus $R(X / R(X))=0$. Finally $X \in \mathscr{B}^{r}$ iff there is no $(B \rightarrow X) \neq 0$ with $B \in \mathscr{B}$, hence iff $X$ does not possess non-trivial $\mathscr{B}$-submodules and this is equivalent to $R(X)=0$.

Remarks 1.3. For modules over rings a fourth statement "dual" to (ii) is equivalent to the three statements of the theorem, (14). It reads as follows:

(ii)' $\mathscr{C}$ is closed under monomorphic preimages, products, $\mathscr{C}$-extensions and $\mathscr{B}=\mathscr{C}^{l}$.

Here the implication (i) $\Rightarrow$ (ii)' is certainly true. We assert that (ii)' implies (iii) with the word "idempotent" omitted. To obtain this assertion take $R(X)=$ the intersection of all the normal submodules $K$ of $X$ such that $X / K \in \mathscr{C}$, hence $C=\Pi X / K \in \mathscr{C}$ and the arrow $X \rightarrow C$ determined by the arrows $X \rightarrow X / K$ admits $R(X)$ as kernel. Thus $X / R(X) \rightarrow C$ is monic, so $X / R(X) \in \mathscr{C}$ and $R(X / R(X))=0$ since $\mathscr{C}=\{X \mid R(X)=0\}$. For any $f: X \rightarrow Y$ and any normal submodule $L$ of $Y$ for which $Y / L \in \mathscr{C}$, write $K$ for the kernel of $X \rightarrow Y \rightarrow Y / L$. So $X / K \rightarrow Y / L$ is monic and $X / K \in \mathscr{C}$. But $f(K) \subset L$, hence every $L$ appearing in the intersection which determines $R(Y)$ includes an $f(K)$, thus $f(R(X)) \subset$ $R(Y)$. Finally $\mathscr{C}^{l}=\{X \mid R(X)=X\}$ since $R(X)=X$ iff there is no $K \neq X$ with $X \mid K \in \mathscr{C}$, so iff there is non-zero $X \rightarrow C \in \mathscr{C}$.

Observe that the assumption $(a)$ was not employed. Observe further that an assumption of the form "subnormal is normal" would lead to idempotence $R^{2}=R$, hence to the equivalence of (i), (ii)', (iii). Indeed, if $R(R(X)) \neq R(X)$ then there is a non-zero $R(X) / K$ in $\mathscr{C}$ and if $K \triangleleft X$ we are done since $X / R(X)$ and $R(X) / K$ in $\mathscr{C}$ imply $X / K \in \mathscr{C}$, so $R(X) \subset K$. We summarise the remarks in the following proposition.

Proposition 1.4. For classes $\mathscr{B}, \mathscr{C}$ the implication (i) $\Rightarrow$ (ii)' $\Rightarrow$ ((iii)-idempotent) holds. If "subnormal is normal" in the category then (i), (ii)', (iii) are equivalent. If (a) also holds then (i), (ii), (ii)', (iii) are equivalent.

Definition 1.5. A pre-torsion theory on $\mathscr{A}$, with (a) assumed, is a pair of classes $\mathscr{B}, \mathscr{C}$ satisfying the equivalent statements of Theorem 1.2.

Thus there is a 1-1 correspondence between pre-torsion theories and idempotent radicals. The objects of the classes $\mathscr{B}, \mathscr{C}$ associated with a radical $R$ will be called $\boldsymbol{R}$-radical, resp. $\boldsymbol{R}$-semisimple.

If (ii), (ii)' are not equivalent, then the two objects $R(X)$ constructed above, namely by "intersections" or say "unions" may not be equal. However, if $R$ constructed by intersections is idempotent then it yields the same objects as the previous $R$ by the following corollary.

Corollary 1.6. Let $R$ be an idempotent radical. Then $R(X)=\cap\left\{K \triangleleft X \mid X / K \in \mathscr{C}_{R}\right\}$.

Proof. Let $L$ be the intersection. Since $X / R(X) \in \mathscr{C}$ it follows that $L \subset R(X)$. Now if $X / K \in \mathscr{C}$ then $(R(X)+K) / K \in \mathscr{C}$. However, $(R(X)+K) / K \cong R(X) /(R(X) \cap K) \in \mathscr{B}$ hence $(R(X)+K) / K=0$. Therefore $R(X) \subset K$ and so $R(X) \subset L$. 
Observe that a sum of submodules appears in the proof. It is not difficult to show that, since the near-ring is distributively generated, a sum of two normal submodules is a normal submodule.

In module theory over rings a radical related to a torsion theory satisfies a condition stronger than $X \subset Y \Rightarrow R(X) \subset R(Y)$. Here we obtain

Theorem 1.7. For classes $\mathscr{B}, \mathscr{C}$ the following are equivalent, assuming (a).

(1) $\mathscr{B}$ is closed under epimorphic images, monomorphic pre-images, extensions, coproducts and $\mathscr{C}=\mathscr{B}$.

(2) There is a radical $R$ (necessarily idempotent) such that $X \triangleleft Y \Rightarrow X \cap R(Y)=R(X)$ and $\mathscr{B}=\{X \mid R(X)=X\}, \mathscr{C}=\{X \mid R(X)=0\}$.

Proof. For $x \in X \cap R(Y)$, the $\bigwedge$-submodule generated by $x$ is in $R(Y)$ so in $\mathscr{B}$, hence in $R(X)$. This completes the implication (1) $\Rightarrow(2)$. If $X \subset Y \in \mathscr{B}$ then $R(Y)=Y$ so $R(X)=X \cap Y=X$ and $X \in \mathscr{B}$. Hence $(2) \Rightarrow(1)$.

Definition 1.8. A radical $R$ satisfying $X \cap R(Y)=R(X)$ for $X \triangleleft Y$ is a torsion radical; $(\mathscr{B}, \mathscr{C})$ satisfying $(1),(2)$ of the theorem is a torsion theory for $(\bigwedge, \Sigma)$-mod.

\section{Extending the torsion and torsion-free functors from $\mathscr{A} b$ to $\mathscr{G} r$}

It is well known that the torsion elements of an abelian group $A$ form a subgroup $t A$ of $A$ characterised as the smallest subgroup $H$ such that $A / H$ is torsion-free. If $A$ is an arbitrary group, the torsion elements do not necessarily form a subgroup and even if we consider the subgroup $t A$ generated by the torsion elements of $A$ then $A / t A$ need not be torsion-free.

We construct a fully-invariant subgroup $T(A)$ of an arbitrary group $\mathrm{A}$, which has exactly the same characterisation as $t A$ in the abelian case.

Definition 2.1. For an arbitrary group $A$ we define inductively $t_{0}(A)=0, t_{1}(A)=$ the subgroup of $A$ generated by the set of torsion elements of $A$ and $t_{k+1}(A) / t_{k}(A)=$ $t_{1}\left(A / t_{k}(A)\right)$. Finally denote $T(A)=\cup_{k} t_{k}(A)$. We call $A$ a pre-torsion group if $T(A)=A$.

Theorem 2.2. (1) $A / T(A)$ is torsion-free. (2) If $H \triangleleft A$ such that $A / H$ is torsion-free then $H \supset T(A)$.

Proof. If $x+T(A)$ is of finite order $m$ then $m x$ belongs to some $t_{k}(A)$ so $x \in$ $t_{k+1}(A) \subset T(A)$. Now if $A / H$ is torsion-free and $T(A) \not \subset H$ then there is a $k \geqq 0$ with $t_{k}(A) \subset H$ and $t_{k+1}(A) \not \subset H$. Let $x \in t_{k+1}(A) \backslash H, x=x_{1}+\ldots+x_{r}$ with integers $m_{1}, \ldots, m_{r}$ such that $m_{j} x_{j} \in t_{k}(A)$ for $j=1, \ldots, r$. At least one of the $x_{j}$ 's is not in $H$, yet $m_{j} x_{j} \in H$. A contradiction.

It may be shown that $T(A)=\cap\{\operatorname{ker} f \mid f: A \rightarrow X, X$ torsion-free $\}$.

Theorem 2.3. $T$ is an idempotent radical on $(Z,\{1\})$-mod with $\mathscr{B}=$ the class of pre-torsion groups and $\mathscr{C}=$ the class of torsion-free groups. $(\mathscr{B}, \mathscr{C})$ is a pre-torsion theory. 
Proof. $\mathscr{B}=\mathscr{C}^{l}$ and $\mathscr{C}=\mathscr{B}^{r}$.

The functors $t_{k}$ are idempotent pre-radicals for all $k$. If we regard them as functors from $\mathscr{G}_{r}$ to the subcategory of groups with $t_{k}(A)=A$, then they are adjoint to the respective inclusion functors. However, for $T$ we obtain more.

Corollary 2.4. The following are adjoint pairs of functors:

(i) I/T: groups $\rightarrow$ torsion-free groups, inclusion: torsion-free groups $\rightarrow$ groups;

(ii) inclusion: pre-torsion groups $\rightarrow$ groups, $T:$ groups $\rightarrow$ pre-torsion groups.

The above is an extension of well-known facts in $\mathscr{A} b$ since pre-torsion-abelian is identical to torsion abelian.

Corollary 2.5. If $A$ is a pre-torsion group then $A / A^{\prime}$ is a torsion group, $A^{\prime}$ the derived group of $A$.

Proof. Every epimorphic image of $A$ is a pre-torsion and $A / A^{\prime}$ is abelian.

Remark 2.6. An abelian group is a torsion group iff $\operatorname{hom}(A, \boldsymbol{Q})=0, \boldsymbol{Q}$ the additive group of rationals. In the general case if $T(A)=A$ then $\operatorname{hom}(A, \boldsymbol{Q})=0$. The converse is not true: if $A$ is a simple torsion-free group then $T(A)=0=\operatorname{hom}(A, Q)$. Such groups are constructed, for instance, in (7).

A group $A$ will be called a torsion-generated group if $t_{1}(A)=A$.

2.7. A subgroup of a torsion generated group may not be, but a quotient group is, torsion-generated. Any coproduct of torsion-generated groups is torsion-generated.

2.8. Every group is embeddable into a symmetric group, yet this latter group is torsion-generated. Moreover, every permutation on a finite or infinite number of letters is the product of two permutations of order 2, (24). The same applies to the alternating groups.

Proposition 2.9. The special linear groups $S L(n, Z)$, and $S L(n, F)$ for fields $F$, are torsion-generated. The orthogonal groups $0(n)$ are torsion-generated.

Proof. In (11) it is shown that, for $n \geqq 2, S L(n, Z)$ is generated by the matrices

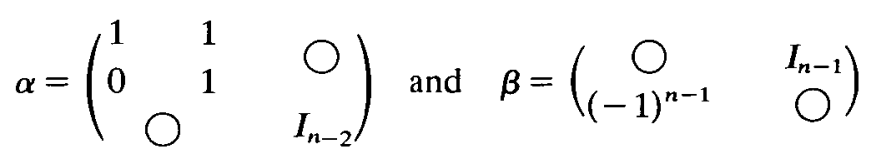

It may be verified that $\beta$ is a torsion element $\left(\beta^{n}=1\right.$ for $n$ odd, $\beta^{2 n}=1$ for $n$ even). Now, with

$$
\gamma=\left(\begin{array}{rrr}
1 & -1 & \bigcirc \\
1 & 0 & \\
& \bigcirc & I_{n-2}
\end{array}\right) \text { and } \delta=\left(\begin{array}{rrr}
0 & 1 & \bigcirc \\
-1 & 0 & \\
\bigcirc & & I_{n-2}
\end{array}\right)
$$

we obtain $\alpha=\gamma \delta$ and $\gamma^{4}=1, \delta^{6}=1$. So $S L(n, Z), n \geqq 2$, is generated by the torsion elements $\beta, \gamma, \delta$. 
To prove the assertion for $S L(n, F)$ observe that it is generated by transvections (24). Every transvection is a conjugate of $I_{n}+E_{1 n} \in S L(n, Z)$. Hence the conjugates of $\beta, \gamma, \delta$ in $G L(n, F)$ generate $S L(n, F)$. (For $n \geqq 3$ it suffices to take the conjugates in $S L(n, F)$.)

For $0(n)$ the assertion follows from the canonical form of an orthogonal matrix and the obvious identity

$$
\left(\begin{array}{cc}
\cos \theta & \sin \theta \\
-\sin \theta & \cos \theta
\end{array}\right)=\left(\begin{array}{cc}
\cos \frac{\theta}{2} & \sin \frac{\theta}{2} \\
\sin \frac{\theta}{2} & -\cos \frac{\theta}{2}
\end{array}\right)\left(\begin{array}{cc}
\cos \frac{3 \theta}{2} & \sin \frac{3 \theta}{2} \\
\sin \frac{3 \theta}{2} & -\cos \frac{3 \theta}{2}
\end{array}\right)
$$

The general linear groups are obviously not torsion-generated, but we will see that $T$ coincides with $t_{1}$ for them.

Corollary 2.10. Let $K$ be either a field $F$ or the ring $Z$. Then

$$
T(G L(n, K))=t_{1}(G L(n, K))=\left\{\alpha \mid \exists r, \alpha^{r} \in S L(n, K)\right\} \text {. }
$$

Proof. If $\alpha \in t_{1}\left(G L_{n}\right)$ then $\alpha=\beta_{1} \ldots \beta_{k}$ and $\beta_{j}^{j}=1$ for some $r_{j}$ 's. Put $r=\Pi r_{j}$, so $\operatorname{det}\left(\alpha^{r}\right)=(\operatorname{det} \alpha)^{r}=1$, thus $\alpha^{r} \in S L_{n}$. Conversely, assume $\alpha^{r} \in S L_{n}$. Write $a$ for $\operatorname{det} \alpha$, so

$$
\left(\begin{array}{cc}
a^{r-1} & \bigcirc \\
\bigcirc & I_{n-1}
\end{array}\right) \alpha=\beta \in S L_{n},
$$

since det $\beta=a^{r-1} a=1$. The matrix $\beta$ is a product of elements of finite order, and

$$
\left(\begin{array}{cc}
a^{r-1} & \bigcirc \\
\bigcirc & I_{n-1}
\end{array}\right)
$$

is of finite order, so $\alpha \in t_{1}\left(G L_{n}\right)$. Finally $T\left(G L_{n}\right)=t_{1}\left(G L_{n}\right)$, for if $\alpha^{s} \in t_{1}\left(G L_{n}\right)$ then there is a $k$ such that $(\operatorname{det} \alpha)^{s k}=1$, i.e., $\alpha^{s k} \in S L_{n}$.

The following is another important class of groups with $T=t_{1}$. It is well known (9), (24) that the torsion elements of a nilpotent group form a subgroup, hence

Proposition 2.11. If $A$ is nilpotent then $T(A)=t_{1}(A)$.

So far we only demonstrated groups for which $T$ is $t_{1}$. The following shows that this is not true in general.

Proposition 2.12. The following group

$$
A=\left\langle x, y ; x^{2}=1,\left(x y^{2}\right)^{2}=1\right\rangle
$$

is not torsion-generated and $t_{2}(A)=A$.

Proof. In $A, x y^{k}=y^{-2} x y^{k-2}$ for $k \geqq 2$ and $x y^{-k}=y^{2} x y^{-k+2}$ for $k \geqq 1$, so every element $a$ in $A$ can be written (even uniquely - as it can be shown) in one of the two forms

$$
a_{(1)}=y^{m}(x y)^{r} \text { or } a_{(2)}=y^{m}(x y)^{r} x
$$

with $r \geqq 0$. We claim that if $m+r$ is odd then $a$ is not of finite order. To prove this, we employ the infinite dihedral group $D=\left\langle x, y ; x^{2}=(x y)^{2}=1\right\rangle$ and a coproduct of two 
two-element groups $H=\left\langle x, y ; x^{L}=y^{L}=1\right\rangle$. Both $D$ and $H$ are epimorphic images of $A$, hence if $a$ is of finite order in $A$ it must be of finite order in $D$ and in $H$. Now, if $m$ is even then $r$ is odd and $a_{(1)}$ in $H$ is $(x y)^{r}$ which is not of finite order, $a_{(2)}$ in $D$ is $y^{m-1}$ not of finite order. If $m$ is odd then $r$ is even and $a_{(1)}$ in $D$ is $y^{m}, a_{(2)}$ in $H$ is $(y x)^{r+1}$, both not of finite order. Now $y \notin t_{1}(A)$ since the contrary implies $y=w_{1} \ldots w_{k}$ with all elements $w_{j}$ of finite order; but at least one $w_{j}$ must contain an odd number of $y$ 's to yield $y$ as their product, a contradiction. Evidently $y^{2}=x\left(x y^{2}\right) \in t_{1}(A)$ so $y \in t_{2}(A)$, hence $A=t_{2}(A)$.

2.13. Consider the following sequence of groups

$$
A_{n}=\left\langle x_{1}, \ldots, x_{n} ;\left(x_{1}\left(x_{2}\left(\ldots\left(x_{i-1} x_{i}^{2}\right)^{2} \ldots\right)^{2}\right)^{2}=1,1 \leqq i \leqq n\right\rangle\right.
$$

for all $n \in N$, and $A_{\omega}=\cup_{n} A_{n}$. This sequence of groups might provide examples that show that

$$
\begin{aligned}
& t_{n}\left(A_{n}\right)=A_{n} \neq t_{n-1}\left(A_{n}\right) \text { for all } n \in N, \\
& T\left(A_{\omega}\right)=A_{\omega} \neq t_{k}\left(A_{\omega}\right) \text { for all } k \in N .
\end{aligned}
$$

Yet, a good induction process has to be produced to verify the assertions.

Once the above is established, then large classes of groups with $T$ being precisely $t_{n}$ can be produced, for instance using 2.15 or 2.16 .

2.14. Observe that all $t_{n}$ preserve direct products, namely: for any family of groups

$$
t_{n}\left(X B_{\iota}\right)=X_{\iota} t_{n}\left(B_{\iota}\right), 0 \leqq n \leqq \omega,
$$

$\boldsymbol{t}_{\boldsymbol{\omega}}$ denoting $T$. This formula is almost evident for $n=1$, is implied by an easy induction for $n<\omega$, and for $\omega$ since $X$ commutes with direct limits.

In particular a direct product of pre-torsion, resp. torsion-generated, groups is pre-torsion, resp. torsion-generated. Moreover the following is an easy consequence of the formula.

Proposition 2.15. For any family of groups, if $T\left(B_{\iota}\right)$ is precisely $t_{n_{\iota}}\left(B_{\iota}\right), 0 \leqq n_{\iota} \leqq \omega$, then $T$ of the direct product is precisely

$$
T\left(\underset{\iota}{X} B_{\iota}\right)=t_{m}\left(\underset{\iota}{X} B_{\iota}\right), \text { with } m=\sup n_{\iota}
$$

Evidently, the formula of 2.14 does not hold in the general case for products. Actually $T:$ groups $\rightarrow$ pre-torsion groups, as a right adjoint, 2.4 , preserves "products" but the product in the category of pre-torsion groups is not the usual product. However the "dual" holds for $I / T$ which, as a left adjoint, preserves coproducts namely $\amalg B J T\left(U B_{\iota}\right)=$ $\amalg\left(B / T\left(B_{\imath}\right)\right)$.

Proposition 2.16. For a family of groups, if $T\left(B_{\iota}\right)$ is precisely $t_{n_{\iota}}\left(B_{\iota}\right), 0 \leqq n_{\iota} \leqq \omega$, then

$$
T\left(\underset{\iota}{\amalg} B_{\iota}\right)=t_{m}\left(\underset{\iota}{\amalg} B_{\iota}\right), \text { with } m=\sup n_{\iota} .
$$


Proof. $T\left(B_{\iota}\right)=t_{m}\left(B_{\iota}\right)$ for all $\iota$ and $\amalg\left(B_{\downarrow} / t_{m}\left(B_{\iota}\right)\right)$ is torsion-free; so $\amalg B_{\downarrow} t_{m}\left(\amalg B_{\iota}\right)$ is torsion-free, since $t_{m}\left(\amalg B_{\iota}\right)$ certainly contains the normal closure $\left[\amalg t_{m}\left(B_{\iota}\right)\right]$ and $B\lrcorner \amalg\left[t_{m}\left(B_{\imath}\right)\right] \cong \amalg\left(B_{\jmath} / t_{m}\left(B_{\mathfrak{\imath}}\right)\right)$.

The group of 2.12 is, in a certain sense, a "minimal example" by the following

Theorem 2.17. For à one-relator group $A, T(A)=t_{1}(A)$. If $A$ is a direct product or $a$ coproduct of one-relator groups, then $T(A)=t_{1}(A)$.

Proof. The single relation on $A$ is of the form $w^{k}$, with the word $w$ not a true power. Then $t_{1}(A)$ is the normal subgroup generated by $w$. It follows that $A / t_{1}(A)$ is a one-relator group with the relator $w$ and it is torsion-free (see (18)). The second assertion follows from the last propositions.

Observe that the last proposition may be of importance in presentation theory where one sometimes looks for conditions which determine whether or not a given group is a one-relator group. The radical $T$ provides such a condition; namely, if $T(A) \neq t_{1}(A)$ then $A$ is not a one-relator group and even not a direct product or a coproduct of one-relator groups.

2.18. Assume $T(B)=t_{k}(B)$ and $N \triangleleft B$. Is $T(B / N)=t_{k+1}(B / N)$ ? 2.12 supplies us with a counter-example. Take $B=\left\langle x, y ; x^{-1} y^{2} x y^{2}=1\right\rangle$ and $N$ the normal subgroup of $B$ generated by $x^{2}$. Then $B$ is torsion-free and $B / N$ is $A$ of 2.12 . Thus $T(B)=t_{0}(B)$ and $T(B / N)=t_{2}(B / N)$. With 2.13 established we could produce, for any $k$, groups $B, N$ with $T(B / N)=t_{k}(B / N)$ and $B$ torsion-free.

\section{Other radicals on $\mathscr{G r}$}

Evidently a class of $(Z,\{1\})$-modules satisfying $1.7(1)$ is either trivial or the whole category. This implies

Corollary 3.1. There is no non-trivial torsion theory on the category of groups.

Consider Nil-c, the category of groups of nilpotence class $c$. This category admits coproducts ( $\amalg A_{\iota}$ in Nil-c is the quotient group of the ordinary free-product of the $A_{\iota}$ 's over the group generated by commutators of the form $\left.\left[x_{1}, \ldots, x_{c+1}\right]\right)$. So any pre-torsion theory is given by classes $\mathscr{B}, \mathscr{C}$ as in 1.2 . For nilpotent groups $N$, the elements of finite order form a subgroup, $t(N)=t_{1}(N)$, hence the radical $T$ induces a torsion theory on Nil-c.

3.2. Denote by $d_{1}(A)$ the product of all normal divisible subgroups of a group $A$ and inductively $d_{\alpha+1}(A) / d_{\alpha}(A)=d_{1}\left(A / d_{\alpha}(A)\right), d_{\alpha}(A)=\bigcup_{\iota<\alpha} d_{\iota}(A)$ for limit ordinals. Finally $D(A)=d_{\alpha}(A)$ if $\alpha$ is the first ordinal for which $d_{\alpha}(A)=d_{\alpha+1}(A)$.

$D$ is an idempotent radical. The $D$-radical groups are those groups all of whose non-trivial epimorphic images contain a non-trivial divisible subgroup. " $D$-semisimple group" is synonymous to "reduced group".

3.3. Kurosh and Chernikov have introduced the class of $R K$-groups (13). These groups are precisely the semisimple groups for the following radical $\delta$. Denote by $\delta_{\alpha}(A)$ 
the $\alpha$-th term of the derived series for $A$ and $\delta(A)=\delta_{\alpha}(A)$, the first ordinal for which $\delta_{\alpha}(A)=\delta_{\alpha+1}(A)$. The $\delta$-radical groups are the perfect groups.

3.4. Denote by $\Gamma_{\alpha}(A)$ the $\alpha$-th term of the lower central series of a group $A$ and $\Gamma(A)=\Gamma_{\alpha}(A), \alpha$ the first ordinal for which $\Gamma_{\alpha}(A)=\Gamma_{\alpha+1}(A)$. Each $\Gamma_{\alpha}$ is a non-idempotent radical and $\Gamma$ is an idempotent radical. " $\Gamma_{\alpha}$-semisimple" is "nilpotent of class $\leqq \alpha ", ~ " \Gamma_{\alpha}$ (or $\Gamma$ )-radical" is "perfect". The $\Gamma$-semisimple class consists of all groups $A$ for which $\Gamma_{\nu}(A)=0$ for some ordinal $\nu$. Kurosh and Chernikov (13) called this the class of $Z D$-groups.

3.5. Both the above examples may be generalised as follows. Let $F$ be a free group and $W$ a set of words in $F$. For any group $A$ denote by $R(A)$ the normal subgroup of $A$ generated by all the words of $W$ in $A$ (i.e. all images of $W$ for any homomorphism $F \rightarrow A$ ). $R$ is a radical with semisimple class = the variety of groups satisfying the set of laws $W$.

Consider again $\delta_{1}$. This is a radical but not idempotent. $\mathscr{B}$ is still the class of perfect groups and $\mathscr{C}$ is the class of abelian groups. $\mathscr{B}=\mathscr{C}^{l}$ but $\mathscr{C} \neq \mathscr{B}^{r}=$ the class of $R K$-groups. This is a particular case of the following proposition which is a weak form of 1.2.

Proposition 3.6. For a radical $R$ with $\mathscr{B}, \mathscr{C}$ as in 1 the following holds.

(i) $\mathscr{B}=\mathscr{C}^{\prime}$;

(ii) $\mathscr{B}$ is closed under epimorphic images, extensions, coproducts;

(iii) $\mathscr{C}$ is closed under monomorphic preimages and products.

\section{The class of radicals and the class of pre-radicals}

We summarise some facts about classes of radicals and pre-radicals. Proofs of results presented here will appear in a future paper (3).

We denote by $\mathscr{R}$ ad the class of radicals on $(\bigwedge, \Sigma)$-mod. (We assume (a).) $\mathscr{R}$ ad is a monoid with respect to composition of functors.

Each radical $R$ determines $\mathscr{B}_{R}$ (=the radical objects) and $\mathscr{C}_{R}$ (=the semisimple objects). We denote $\mathscr{C}=\left\{\mathscr{C}_{R} \mid R \in \mathscr{R}\right.$ ad $\}$. We employ a common construction in varieties (19), (21): if $R, S \in \mathscr{R}$ ad we denote by $\mathscr{C}_{R} \circ \mathscr{C}_{S}$ the collection of $(\bigwedge, \Sigma)$-modules $A$ such that there is a normal submodule $A^{\prime}$ in $A$ with $A^{\prime} \in \mathscr{C}_{R}$ and $A / A^{\prime} \in \mathscr{C}_{S}$. Then $\langle\mathscr{C}, \circ\rangle$ is a monoid and it includes the collection of varieties as a submonoid. The mapping $R \mapsto \mathscr{C}_{R}$ is an epimorphism of monoids.

The intersection of any set of radicals, namely $R=\cap R_{\imath}$ defined by $R(A)=\cap R_{\iota}(A)$ for all $A$, is a radical. This fact yields a simple method of constructing an idempotent radical $\bar{R}$ from a given radical $R$. For ordinals $\iota$, let $R^{\iota+1}=R \circ R^{\iota}$ and $R^{\iota}=\bigcap_{\nu<\iota} R^{\nu}$ for limit ordinals. Then $R^{\iota} \in \mathscr{R}$ ad for all $\iota$ and we denote $\bar{R}=R^{\iota}, \iota$ the first-ordinal such that $R^{\iota}=R^{\imath+1}$. (The example 3.3 fits this construction with $R=\delta_{1}$.)

The following is implied.

Proposition 4.1. $\quad \bar{R}$ is an idempotent radical with $\mathscr{B}_{\bar{R}}=\mathscr{B}_{R}$.

We denote by $p \mathscr{R}$ ad the class of pre-radicals on $(\bigwedge, \Sigma)$-mod. The following is a weak form of $1.2+1.3$. 
Theorem 4.2. For an idempotent pre-radical (i) $\mathscr{B}^{r}=\mathscr{C}$; (ii) $\mathscr{B}$ is closed under epimorphic images and coproducts; (iii) $\mathscr{C}$ is closed under monomorphic preimages, products and extensions. Even without "idempotent" the inclusion $\mathscr{B} \subset \mathscr{C}^{\prime}$ holds.

Let $B=\left\{\mathscr{B}_{R} \mid R \in p \mathscr{R}\right.$ ad $\}$. It follows that $\langle p \mathscr{R}$ ad,o $\rangle$ and $\langle B, \cap\rangle$ are isomorphic monoids under the map $R \mapsto \mathscr{B}_{R}$.

For $R, S \in p \mathscr{R a d}$ we define $R \times S$ by $(R \times S)(A) / S(A)=R(A / S(A))$. A-straightforward argument shows that $R \times S$ is a pre-radical.

Here is a method of constructing a radical $\tilde{R}$ from a given pre-radical $R$. For ordinals $\iota$ put $R_{\iota+1}(A)=R \times R_{\iota}(A)$ and $R_{\iota}(A)=\bigcup_{\nu<\iota} R_{\nu}(A)$ for a limit ordinal. Finally $\tilde{R}(A)=R_{\iota}(A)$ for the first $\iota$ with $R_{\iota+1}(A)=R_{\iota}(A)$. The following is readily established.

Proposition 4.3. $\tilde{R}$ is a radical. If $R$ is idempotent then $\tilde{R}$ is idempotent and $\mathscr{C}_{\tilde{R}}=\mathscr{C}_{R_{t}}$ for all $\iota$.

The radical $T$ on $\mathscr{G}_{r}$, Section 2 , may be represented in this way. Start with $t_{1}$ which is clearly an idempotent pre-radical and $\tilde{t}_{1}=t_{\omega}=T$. Observe that $\mathscr{B}_{T}$ is the class of groups whose every non-trivial epimorphic image possess a non-trivial torsion subgroup. A similar fact holds for the radical $D$ of 3.2. This suggests the following. With any object function $R$ and $\mathscr{B}_{R}$ call an object $A$ a hyper-R-object if every non-trivial epimorphic image of $A$ possesses a non-trivial $\mathscr{B}_{R}$-subobject. Then

Proposition 4.4. Let $R$ be an idempotent pre-radical and $\tilde{R}$ as constructed above. Then $\mathscr{B}_{\bar{R}}$ is the class of hyper- $R$-objects.

Here are some additional results.

Proposition 4.5. (i) Let $R$ be an idempotent pre-radical. Then $\mathscr{B}_{R_{n}} \circ \mathscr{B}_{R_{m}} \subset \mathscr{B}_{R_{n+m}}$ for all non-negative integers $n, m$. For $\mathscr{C}$ a similar fact holds even without idempotence. (ii) Let $R$ be a pre-radical. Then $\mathscr{C}_{R^{n}} \circ \mathscr{C}_{R^{m}} \subset \mathscr{C}_{R^{n+m}}$ for all non-negative integers $n, m$.

A well-known example for the radicals in 3.5. If $H \triangleleft A, H$ nilpotent of class $\leqq n, A / H$ nilpotent of class $\leqq m$, then $A$ is nilpotent of class $\leqq n+m$.

The following are preliminary remarks concerning series of submodules. $R$ stands for an object function on $(\bigwedge, \Sigma)$-modules.

An ascending $R$-series for $A$ is a sequence of normal $\wedge$-submodules $0 \triangleleft A_{1} \triangleleft \ldots \triangleleft A_{\alpha}=A$ such that $A_{\beta+1} / A_{\beta} \in \mathscr{B}_{R}$ for every index ordinal $\beta$ and $A_{\beta}=\underset{\nu<\beta}{\cup} A_{\nu}$ for every limit ordinal $\beta$. A.descending $R$-series for $A$ is a sequence of normal $\Lambda$ submodules $0=A_{\alpha} \triangleleft \ldots \triangleleft A_{1} \triangleleft A$ such that $A_{\beta} / A_{\beta+1} \in \mathscr{C}_{R}$ for every index ordinal $\beta$ and $A_{\beta}=\bigcap_{\nu<\beta} A_{\nu}$ for limit ordinal $\beta$.

The following facts are obtained. 
Proposition 4.6. Let $R$ be an idempotent pre-radical. Then $A \in \mathscr{B}_{\bar{R}}$ iff there exists an ascending $R$-series for $A$. In this case the sequence $0 \triangleleft R(A) \ldots \triangleleft \tilde{R}(A)=A$ is the unique upper $R$-series for $A$.

Corollary 4.7. For any class $\mathscr{D}$ put poly-D for the class of $(\bigwedge, \Sigma)$-modules A having a finite series $0=A_{0} \triangleleft A_{1} \triangleleft \ldots \triangleleft A_{n}=A$ with $A_{i} / A_{i-1} \in \mathscr{D}$ for $1 \leqq i \leqq n$. Then, for an idempotent pre-radical $R$, poly $\mathscr{B}_{R}=\mathscr{B}_{R_{\omega}}$.

Proposition 4.8. Let $R$ be a radical. Then $A \in \mathscr{C}_{R}$ iff there exists a descending $R$-series for $A$. In this case the series $0=\bar{R}(A) \triangleleft \ldots \triangleleft R(A)=A$ is the unique lower $R$-series for $A$.

\section{REFERENCES}

(1) M. BARR, Non-abelian torsion theories, Can J. Math. 25 (1973), 1224-1237.

(2) S. E. Dickson, A torsion theory for abelian categories, Trans. Amer. Math. Soc. 121 (1966), 223-235.

(3) S. FEIGELSTOCK and A. KLEIN, Functorial radicals and non-abelian torsion, II (in preparation).

(4) A. FRÖHLICH, On groups over a d.g. near-ring, I, II, Quart. J. Math. 11 (1960), 193-228.

(5) O. Goldman, Rings and modules of quotients, J. Algebra 13 (1969), $10-47$.

(6) P. Hall, On Frattini subgroups of finitely-generated groups, J. London Math. Soc. 24 (1949), 353-384.

(7) G. Higman, B. H. NEUMANN and H. NeUMANN, Embedding theorems for groups, $J$. London Math. Soc. 24 (1949), 247-254.

(8) P. J. Huton, Localization and cohomology of nilpotent groups, Math. Zeit. 132 (1973), 263-286.

(9) K. HirSCH, On infinite soluble groups II, Proc. London Math. Soc. (2) 44 (1938), 336-344.

(10) M. Holcombe and R. WAlker, Radicals in categories, Proc. Edinburgh Math. Soc. 21 (1978), 111-128.

(11) R. A. KatZ and W. Magnus, Residual properties of free groups, Comm. Pure \& Appl. Math. 22 (1969), 1-13.

(12) A. KleIN, Injectives and simple objects, J. Pure App. Algebra 15 (1979), 243-245.

(13) A. G. KUROSH and S. N. CHERNIKOv, Solvable and nilpotent groups, Uspehi 2-3 (1947), 18-59.

(14) J. LAMBEK, Torsion theories, additive semantics and rings of quotients (Lecture Notes 177, Springer, 1971).

(15) J. LAMBEK and B. RATTRAY, Localization and codensity triples, Comm. Alg. 1 (1974), 145-164.

(16) J. LAMBEK and B. RATTRAY, Localization and sheaf reflectors. Trans. Amer. Math. Soc. 210 (1975), 279-293.

(17) J. LAMBEK and B. RATTRAY, Localization and duality in additive categories, Houston J. Math. 1 (1975), 87-100. 
(18) W. MAGnus, A. Karrass and D. SOlTTAR, Combinatorial Group Theory (Interscience, New York and London, 1966).

(19) J. D. P. MELDRUM, The group d.g. near-ring, Proc. London Math. Soc. 32 (1976), 323-346.

(20) H. NEUMANN, Varieties of Groups (Springer Verlag, New York, 1967).

(21) B. I. Plotkin, On the nil-radical of a group, Doklady 98 (1954), 341-343.

(22) B. I. PLotKIN, Functorials, radicals, co-radicals, Mat. Zap. Sverdlovsk 7, 3(1970), 150-182.

(23) B. RATTRAY, Torsion theories in non-additive categories, Manuscripta Math. 12 (1974), 285-305.

(24) W. R. SCOTT, Group Theory (Prentice-Hall, N.J., 1974).

(25) R. B. WARFIELD, Jr., Nilpotent groups (Lecture Notes 513, Springer, 1976).

(26) R. WIEgandt, Radicals and semi-simplicity in categories, Acta Math. Ac. Sc. Hung. 19 (3-4) (1968), 345-364.

BAR-ILAN UNIVERSITY

RAMAT-GAN, ISRAEL 